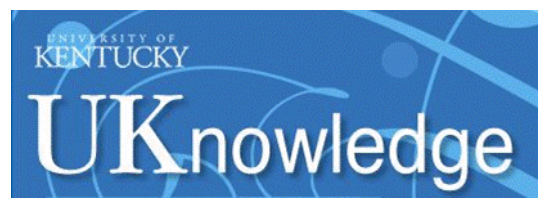

University of Kentucky

UKnowledge

6-20-2016

\title{
Evidence for Fluorescent Fe II Emission from Extended Low Ionization Outflows in Obscured Quasars
}

\author{
Tinggui Wang \\ University of Science and Technology of China, China \\ Gary J. Ferland \\ University of Kentucky, gary@uky.edu \\ Chenwei Yang \\ University of Science and Technology of China, China \\ Huiyuan Wang \\ University of Science and Technology of China, China \\ Shaohua Zhang \\ Polar Research Institute of China, China
}

Follow this and additional works at: https://uknowledge.uky.edu/physastron_facpub

Part of the Astrophysics and Astronomy Commons, and the Physics Commons

Right click to open a feedback form in a new tab to let us know how this document benefits you.

\section{Repository Citation}

Wang, Tinggui; Ferland, Gary J.; Yang, Chenwei; Wang, Huiyuan; and Zhang, Shaohua, "Evidence for Fluorescent Fe II Emission from Extended Low Ionization Outflows in Obscured Quasars" (2016). Physics and Astronomy Faculty Publications. 422.

https://uknowledge.uky.edu/physastron_facpub/422

This Article is brought to you for free and open access by the Physics and Astronomy at UKnowledge. It has been accepted for inclusion in Physics and Astronomy Faculty Publications by an authorized administrator of UKnowledge. For more information, please contact UKnowledge@lsv.uky.edu. 
Evidence for Fluorescent Fe II Emission from Extended Low Ionization Outflows in Obscured Quasars

Digital Object Identifier (DOI)

https://doi.org/10.3847/0004-637X/824/2/106

Notes/Citation Information

Published in The Astrophysical Journal, v. 824, no. 2, 106, p. 1-11.

(C) 2016. The American Astronomical Society. All rights reserved.

The copyright holder has granted the permission for posting the article here.

This article is available at UKnowledge: https://uknowledge.uky.edu/physastron_facpub/422 


\title{
EVIDENCE FOR FLUORESCENT Fe II EMISSION FROM EXTENDED LOW IONIZATION OUTFLOWS IN OBSCURED QUASARS
}

\author{
Tinggui Wang ${ }^{1}$, Gary J. Ferland ${ }^{2}$, Chenwei Yang ${ }^{1}$, Huiyuan Wang ${ }^{1}$, and Shaohua Zhang ${ }^{3}$ \\ ${ }^{1}$ CAS Key Laboratory for Researches in Galaxies and Cosmology, Department of Astronomy, University of Science and Technology of China, Hefei, Anhui 230026, \\ China; twang@ustc.edu.cn \\ 2 Department of Physics and Astronomy, University of Kentucky, Lexington, KY 40506, USA \\ ${ }^{3}$ Polar Research Institute of China, 451 Jinqiao Rd, Shanghai 200136, China \\ Received 2016 January 28; revised 2016 April 7; accepted 2016 April 25; published 2016 June 20
}

\begin{abstract}
Recent studies have shown that outflows in at least some broad absorption line (BAL) quasars are extended well beyond the putative dusty torus. Such outflows should be detectable in obscured quasars. We present four WISE selected infrared red quasars with very strong and peculiar ultraviolet Fe II emission lines: strong UV Fe II UV arising from transitions to ground/low excitation levels, and very weak Fe II at wavelengths longer than $2800 \AA$. The spectra of these quasars display strong resonant emission lines, such as $\mathrm{C}$ IV, $\mathrm{Al}$ III and $\mathrm{Mg}$ II but sometimes, a lack of non-resonant lines such as $\mathrm{C}$ III], $\mathrm{S}$ III and He II. We interpret the Fe II lines as resonantly scattered light from the extended outflows that are viewed nearly edge-on, so that the accretion disk and broad line region are obscured by the dusty torus, while the extended outflows are not. We show that dust free gas exposed to strong radiation longward of $912 \AA$ produces Fe II emission very similar to that observed. The gas is too cool to collisionally excite Fe II lines, accounting for the lack of optical emission. The spectral energy distribution from the UV to the midinfrared can be modeled as emission from a clumpy dusty torus, with UV emission being reflected/scattered light either by the dusty torus or the outflow. Within this scenario, we estimate a minimum covering factor of the outflows from a few to $20 \%$ for the $\mathrm{Fe}$ II scattering region, suggesting that $\mathrm{Fe}$ iI BAL quasars are at a special stage of quasar evolution.
\end{abstract}

Key words: line: formation - quasars: absorption lines - quasars: emission lines - quasars: individual (SDSS J153542.4+090341, SDSS J163246.6+340526, SDSS J100552.6+493448)

\section{INTRODUCTION}

Outflows are ubiquitous among quasars. They manifest themselves as blueshifted broad and narrow absorption lines (NALs) in UV and X-rays (e.g., Boksenberg et al. 1978; Turnshek et al. 1980; Weymann et al. 1991). The broad absorption lines (BALs) with widths of around a few thousands to a few ten thousands $\mathrm{km} \mathrm{s}^{-1}$ appear in $15 \%-25 \%$ of all quasars (e.g.,Weymann et al. 1991; Knigge et al. 2008), and intrinsic NALs with widths around hundreds to one thousand $\mathrm{km} \mathrm{s}^{-1}$ in $40 \%-50 \%$ of quasars (Nestor et al. 2008). Almost all known BAL QSOs show broad high ionization absorption lines, such as C Iv, Nv (Weymann et al. 1991; cf, Mrk 231, Veilleux et al. 2013), while only a small fraction (about $25 \%$ ) of BAL QSOs display also low ionization broad absorption lines (LoBALs, Weymann et al. 1991; Zhang et al. 2010; c.f. Urrutia et al. 2009), commonly Mg II. A yet smaller subset of LoBAL QSOs ( $<1 \%$ of all quasars) exhibit broad Fe II absorption lines (hereafter, FeLoBALs; Trump et al. 2006; c.f., Dai et al. 2012).

There are two different scenarios for BAL and non-BAL quasars or for a subclass of BAL QSOs. In the first scenario, the presence or the absence of BALs, or more generally, the manifestation of different subclasses of BALs, are attributed to the different viewing angles of the same intrinsic population (Weymann et al. 1991). While in the second scenario, LoBAL and non-LoBAL QSOs are at different evolutionary stages (Boroson \& Meyers 1992; Voit et al. 1993; Hall et al. 2002). In other words, only a small fraction of quasars possess lowionization outflows. Early studies showed that the emission lines and broad-band spectral energy distributions (SED) are very similar for HiBAL and non-BAL quasars (Weymann et al.
1991), suggesting the unification scheme. Hamann et al. (1993) constrained the global covering factor to less $20 \%$ from the fraction of resonant scattering light. However, systematic trends in the SED and emission lines have been revealed with a large sample of BAL, LoBAL in particular, and a non-BAL quasar sample from SDSS (e.g., Zhang et al. 2010; Farrah et al. 2010; Baskin et al. 2013; Wang et al. 2013; and reference therein; c.f., Lazarova et al. 2012), suggesting that the global covering factor and properties of LoBALs depend on the intrinsic properties of the system.

Feedback through these outflows is now widely believed to be a key physical process in the formation of massive galaxies (Fabian 2012). However, the mass loss rate and kinetic power, as well as the size, of outflows are still poorly constrained for the majority of BAL quasars (Lucy et al. 2014). To estimate these parameters from absorption lines, one needs to know the global covering factor, column density and the gas density or distance. The gas column density can be inferred from the column densities of a set of ions through photoionization modeling. However, the gas density diagnostics for absorption lines are available for only a rare subset of BAL QSOs with measured absorption lines from low-lying excitation levels of heavy elements (de Kool et al. 2001; Korista et al. 2008; Dunn et al. 2012). Previous measurements of density in a dozen of BALs, mostly FeLoBALs, quasars, indicate that BAL region (BALR) is extended from $100 \mathrm{pc}$ to several $\mathrm{kpc}$ from quasars (de Kool et al. 2001; Korista et al. 2008; Borguet et al. 2013; Chamberlain et al. 2015). The large size implies a very large mass outflow rate $\left(10^{2-3} M_{\odot} \mathrm{yr}^{-1}\right)$ and large kinetic power (a few to $10 \%$ of the total luminosity) if the outflows cover a substantial fraction of the sky. However, absorption lines can 
only probe the gas on the line of sight, so the global covering factor of absorbing gas is virtually unknown in an individual object. Because these quasars are so rare, the average global covering factor must be very small, according to the unification scheme, but if they are at a special evolution stage, on the other hand, the covering factor can be substantial. Interestingly, Liu et al. (2015) detected extended outflow components in two low $z$ active galactic nuclei (AGNs) on the physical sizes consistent with those inferred from ultraviolet absorption lines.

In this paper, we report four quasars, including one that was noted already by Ross et al. (2015), with peculiar ultraviolet Fe II spectra. They were discovered during the course of studying a sample of heavily obscured quasars in WISE: $W 1-W 4>8.0$. We perform an analysis of the emission lines and the broad band SED, and present evidence that the Fe II emission lines in these objects are dominated by continuum fluorescence pumping in the extended outflows, so they can be used to constrain the covering factor and size of BALR of LoFeBAL. The paper is organized as follows. In Section 2, we present an analysis of the optical spectra and broad band SED. In Section 3, we discuss the nature of Fe II emission and continuum emission, and a brief conclusion is given in Section 4. Spectral models of fluorescent Fe II emission are presented in an Appendix.

\section{THE DATA ANALYSIS}

\subsection{The Optical Spectra}

The optical spectra are shown in Figure 1. One of these quasars, SDSS J153542.4+090341 has already been noted in Ross et al. (2015); and another one (SDSS J163246.6+340526) appeared on the list of unusual quasars in Meusinger et al. (2012). The most striking features of these objects are weak continua, strong ultraviolet Fe II spikes and $\mathrm{Mg}$ II emission lines, and a relatively narrow $\mathrm{Mg}$ II line. Individual objects are described and analyzed as follows.

1. SDSS J153542.4+090341: The BOSS spectrum shows three prominent Fe II spikes due to UV1, 2, 3 and 62, 63, and $\mathrm{Mg}$ II, $\mathrm{Al}$ III and $\mathrm{C}$ IV lines. He II 1640+O III1663 and $\mathrm{C}$ III] are weak with respective to these lines. We measure an extreme $\mathrm{Fe}$ II spike/gap ratio ${ }^{4}$ of 8.5. A narrow Fe II 78 is also visible. In fact, the equivalent widths of $\left.\mathrm{C}_{\mathrm{III}}\right]$ and $\mathrm{He}$ II are consistent with those of I Zw 1 (see Figure 1, also Laor et al. 1997), while those of Fe II, $\mathrm{Mg}$ II, $\mathrm{Al}$ III and $\mathrm{C}_{\text {IV }}$ are a factor of 10-20 times larger. This indicates that the UV spectrum consists of two components, a weak I Zw 1 component and another component dominated by strong emission lines, Fe II, $\mathrm{C}_{\mathrm{IV}}, \mathrm{Al}$ III and $\mathrm{Mg}$ II. $\mathrm{C}$ IV shows two components, a narrow component at the systematic redshift, derived from [O II], and a blueshifted component. Alternatively, $\mathrm{C}$ IV may be split into two components due to a blueshifted NAL. The Mg II doublet can be well modeled with two gaussians. The best fit converges to a width of $\mathrm{FWHM}=1230 \pm 60 \mathrm{~km} \mathrm{~s}^{-1}$ and no shift in velocity $\left(2 \pm 500 \mathrm{~km} \mathrm{~s}^{-1}\right)$. [O II] appears fairly narrow overall, but with weak broader wings. [Ne III] $\lambda 3869$ looks similar with a narrow core and extended wings. However,

\footnotetext{
4 It is defined as $\mathrm{Fe}$ II flux over the wavelength ranges 2312-2428 A (hereafter, Fe2B2355) and 2565-2665 A dividing by the total Fe II flux in the range 2462-2530 $\AA$ (Baldwin et al. 2004).
}
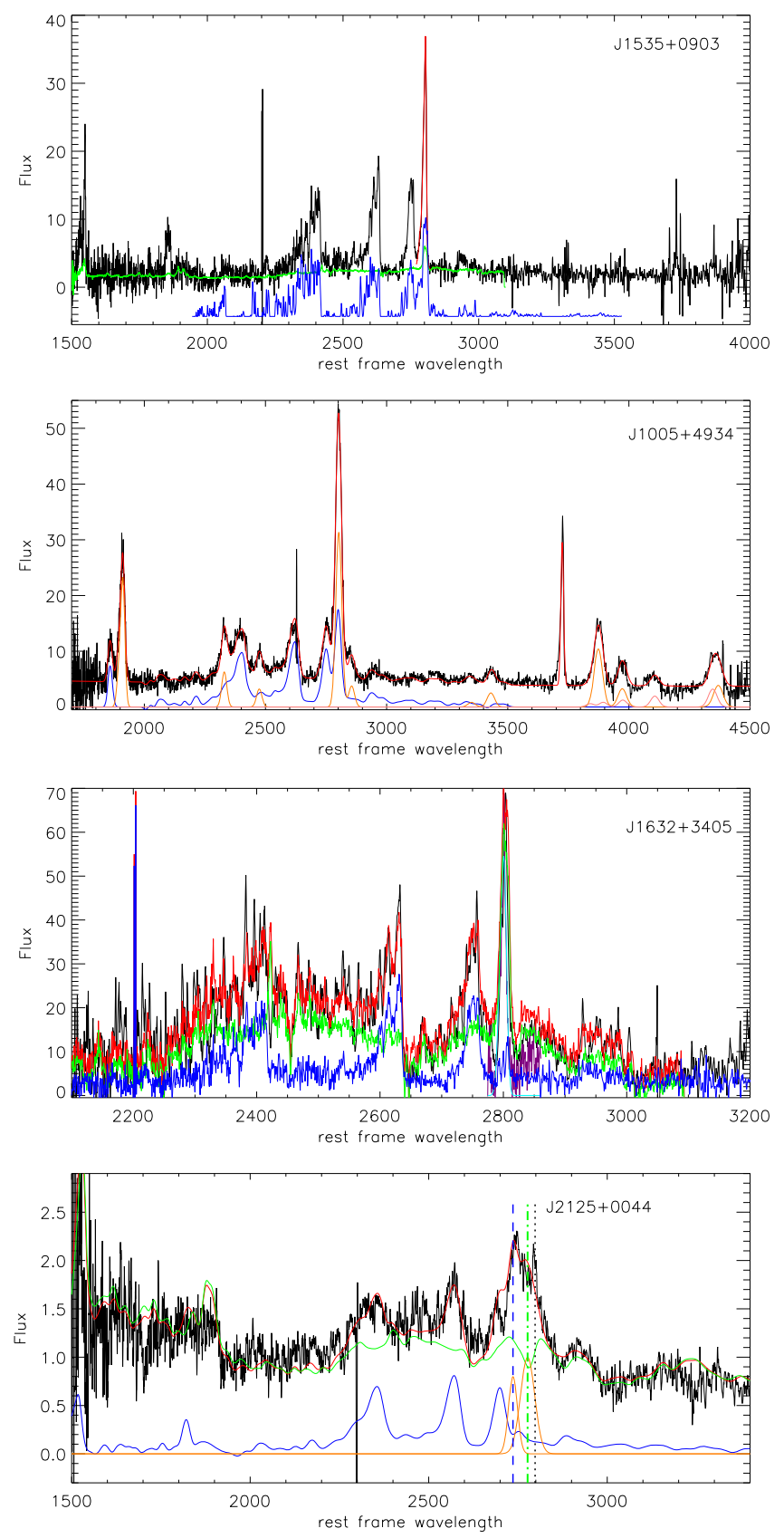

Figure 1. The SDSS spectra of four AGN with peculiar Fe II emission (in black curves). In the top panel, the blue curve is the absorbed spectrum of FeLoBAL QSO SDSS J080957.39+181804.42, which is derived using the best matched composite templates (See Wang et al. 2015 for details). The Fe II spectrum of SDSS J1535+0903 looks remarkably similar to the absorption spectrum of FeLoBAL QSO. In the second panel, the blue curve is the broadened SDSS $\mathrm{J} 1535+0903$ spectrum, while orange curves are gaussians for additional emission lines. In the third panel, the green curve represents the scaled I Zw 1 spectrum, the blue is the scaled SDSS J1535+0903 spectrum, and the red one is the combination of the two. In the bottom panel, colored curves are as in the third panel except that the $\mathrm{Mg}$ II line in each component is replaced by an independent Gaussian. The SDSS J1535+0903 component is blueshifted by $6600 \mathrm{~km} \mathrm{~s}^{-1}$, and the I Zw 1 component by $2300 \mathrm{~km} \mathrm{~s}^{-1}$.

both lines are severely contaminated by the subtraction of sky lines.

2. SDSS J100552.6+493448: In addition to strong ultraviolet Fe II spikes, this object displays very strong highionization forbidden and permitted lines: [O III] $\lambda 4363$, [Ne III] $\lambda \lambda 3869,3987,[\mathrm{Ne} \mathrm{v}] \lambda \lambda 3346,3426,[\mathrm{Ne}$ IV] $\lambda 2422$, 
[C III] $] \lambda 1906,1909$, as well as low ionization lines $\mathrm{H} \gamma, \mathrm{H} \delta$, [OII] $\lambda \lambda 3726,3729, \mathrm{C}$ II] $\lambda 2326, \mathrm{Mg}$ II, and $\mathrm{Al}$ III $\lambda \lambda 1855$, 1863. In fact, the spectrum can be reproduced by a supposition of broadened SDSS J1535+0903 emission lines and the narrow lines mentioned above (See Figure 1). The Al III strength can be well accounted for by the SDSS J1535+0903 component. To first order, all but $[\mathrm{O} \mathrm{II}]$, have the same width, around $\mathrm{FWHM} \simeq 3150 \mathrm{~km} \mathrm{~s}^{-1}$, using a single Gaussian fit. [O II] is considerably narrower than other lines, probably from an extended star-forming region. There is also a weak broad wing in [O II], which may be similar to other lines. The weak line to the red side of $\mathrm{Mg}$ II can be either $\mathrm{Fe}_{\mathrm{II}}$ or $\mathrm{Mg}$ I. [O III] $\lambda 4363$ is stronger than $\mathrm{H} \gamma$ according to the single Gaussian fit with their centers tied to other lines. Unfortunately, the BOSS spectrum does not cover [O III] $\lambda \lambda 4959,5007$, which should impose important constraints on the gas density and gas temperature in combination with $[\mathrm{O}$ III $] \lambda 4363$. Another constraint on the density comes from the fit to 1900 blending. We find that in the single Gaussian model for each line the fit is significantly worse when the lines are assumed to be [Si III] and [C III] with a $\Delta \chi^{2}=30.8$ with respect to models assuming they are $\mathrm{Si}$ III] and $\mathrm{C}$ III]. A further fit with a mixture of $\mathrm{C}$ III] and [C III] yields a [C III]/ $\left.\mathrm{C}_{\text {III }}\right]=0.11 \pm 0.02$ with a $\Delta \chi^{2}=11.8$ lower than pure $\mathrm{C}$ III] model. This gives a gas density (3-4) $\times$ $10^{5} \mathrm{~cm}^{-3}$ (Osterbrock \& Ferland 2006). The outflow has a considerably lower density than that in Q 1321 +058 (Wang et al. 2009). Detailed photoionization modeling of the outflow component will be presented elsewhere.

3. SDSS J163246.6+340526: The spectrum is dominated by narrow ultraviolet Fe II lines. The Fe II spikes/gap ratio (3.2) is less extreme than in $\mathrm{J} 1535+0903$. We adopt the redshift of narrow [O II] as the systemic redshift. Fe II and Mg II appears fairly narrow. We fit the Mg II multiplet using two Gaussian and obtain a best fit with an FWHM $=880 \pm 40 \mathrm{~km} \mathrm{~s}^{-1}$ and a doublet ratio of $I(2796): \mathrm{I}(2803)=0.48 \pm 0.04$ with no systematic redshift. $\mathrm{Mg}$ II is slightly narrower than $\mathrm{Mg}$ II in I Zw 1 (Laor et al. 1997). Interestingly, the observed spectrum in the 2100-3100 A range can be reproduced approximately by the addition of a scaled I Zw 1 spectrum with fourtimes stronger emission lines plus the Fe II spectrum of SDSS J1535+0903 (see Figure 1(c)). [O III] $\lambda 5007$ is visible in the gap of the sky line forest, and has the same redshift as $[\mathrm{O}$ II]. Unfortunately, $\mathrm{H} \beta$ is seriously affected by residuals of sky lines.

4. SDSS J212546.9+004455: Unlike other objects, the continuum emission in this object is relatively strong. The narrow $\mathrm{Mg}$ II line has the same redshift as [O II], which is considered as the systematic redshift. A comparison with the $\mathrm{Fe}$ II template of I $\mathrm{Zw} 1$ and the spectrum of SDSS J1535+0903 suggests the presence of two blueshifted ultraviolet Fe II components. A broadened SDSS J1535+0903-like component is shifted by $5700 \mathrm{~km} \mathrm{~s}^{-1}$ and a I Zw 1-like ultraviolet Fe II component is blueshifted by $\sim 2850 \mathrm{~km} \mathrm{~s}^{-1}$. The I $\mathrm{Zw}$ 1-like component has weaker $\mathrm{Mg}$ II, $\mathrm{C}_{\text {III, }} \mathrm{Si}$ III and $\mathrm{C}$ IV lines but broader line widths than I Zw 1 . We measure the FWHM of Mg II using the above two- component fit (see also Figure 1). There appear NALs of $\mathrm{C}$ IV at velocities of 900 and $2300 \mathrm{~km} \mathrm{~s}^{-1}$. But the signalto-noise ratio is low.

Table 1 presents the emission-line parameters, including the width of $\mathrm{Mg}$ II and the equivalent widths of $\mathrm{Fe}$ II spikes, Mg II and Al III. To summarize, SDSS J1535+0903 represents a prototype peculiar $\mathrm{Fe}$ II spectrum, while the spectra in other objects can be modeled as a combination of this and a $\mathrm{I} \mathrm{Zw}$ 1-like Fe II component.

\subsection{Modeling the SED}

Broad band SEDs of these quasars are shown in Figure 2. They are characterized by strong and steep mid-infrared emission, and a relatively weak ultraviolet and optical continuum. The photometry is strongly affected by the ultraviolet Fe II and $\mathrm{Mg}$ II. We corrected the emission-line flux using the SDSS spectrum. We fit these SEDs using a model consisting of a clumpy torus plus a galaxy template, accounting for the host galaxy light. We use all galaxy templates, excluding AGNs, that are in the SWIRE library (Polletta et al. 2007). The purpose of this fit is to give an estimate how much of the infrared emission comes from the AGN heated dust and how this dust is distributed.

The SEDs of clumpy tori were calculated for a wide range of parameters by Hönig \& Kishimoto (2010). In their models, the torus consists of optically thick clouds with a small total filling factor. The number density of clouds decreases outward in a power law form, and has a Gaussian distribution in the vertical direction. The parameters of the models are the number of clouds along the line of sight on the equator $\left(N_{0}\right)$, the radial dust-cloud distribution index $(\alpha)$, the subtending angle of the torus $\left(\theta_{0}\right)$, the optical depth of individual clouds, and the inclination (refer to Hönig \& Kishimoto 2010 for details). The inner radius is fixed to the dust sublimation radius and the outer radius of the torus to 300 dust sublimation radii.

The broad-band SED can be fitted fairly well with such a model. The best fits are shown in Figure 2 and the parameters are listed in Table 1. We also compute the distribution of the AGN fraction in the rest frame 3-15 $\mu \mathrm{m}$ using all models weighted by the $\chi^{2}$ probability of each model and by the solid angle of the viewing angle, i.e., $w=P\left(\chi^{2}\right.$, dof $) \cos i \Delta i$. The results suggest that the infrared emission is dominated by a torus component, and that the galaxy component contributes less than $5 \%$ in all cases. The power-law index for the dust distribution is quite broad from $-2.0 \geqslant \alpha \geqslant-0.7$. Thus the steepness in the mid-infrared spectrum is due to weak host galaxy light in the near-infrared, indicating a high Eddington ratio of the system, and an edge-on viewing angle. This result is probably a color selection effect, where we only choose objects with a very red infrared color. Second, the half opening angle of the torus is small $\left(45^{\circ}-30^{\circ}\right)$, and the system is viewed at a relatively large inclination angle $\left(65^{\circ}-75^{\circ}\right)$, consistent with the large infrared to optical ratio. Finally, torus models predict a UV flux higher than the observed ones if there is no internal extinction. The reddening to the reflected light can be due to diffuse interstellar dust further out in the host galaxy, which may be partially covering. Note that our model does not include other optical or UV continuum sources, such as direct or electron scattered accretion disk light. If such components are present, then the contribution from the host galaxy should be 
Table 1

Emission Line Measurements

\begin{tabular}{|c|c|c|c|c|c|c|c|c|}
\hline SDSS J & $z$ & $\begin{array}{l}\text { FWHM(Mg II }) \\
\quad\left(\mathrm{km} \mathrm{s}^{-1}\right)\end{array}$ & $\begin{array}{c}\mathrm{W}(\mathrm{Fe} 2 \mathrm{~B} 2355) \\
(\AA)\end{array}$ & $R_{\text {spike }}$ & 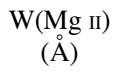 & $\begin{array}{c}\text { W(Al III) } \\
(\AA)\end{array}$ & $\begin{array}{c}\mathrm{W}(\mathrm{C} \text { III }) \\
(\AA)\end{array}$ & $\begin{array}{c}\mathrm{W}(\mathrm{C} \text { IV }) \\
(\AA)\end{array}$ \\
\hline $100552.6+493448$ & 1.1217 & 3150 & 173 & 6.4 & 310 & 35 & 103 & $\ldots^{\mathrm{a}}$ \\
\hline $153542.4+090341$ & 1.5330 & 1230 & 545 & 8.5 & 782 & 186 & 49 & 330 \\
\hline $163246.6+340526$ & 0.8306 & 880 & 280 & 3.2 & 140 & $\ldots$ & $\ldots$ & $\ldots$ \\
\hline $212546.9+004455$ & 1.4240 & 8300 & 82 & 2.9 & 103 & 3 & 6 & 2 \\
\hline
\end{tabular}

Note.

a Typical errorbars for strong lines are $20 \%$ for equivalent widths and 15\% for FWHM, except for J2125+0044, where line width and equivalent width of Mg II are poorly determined due to blending with $\mathrm{Fe}$ II lines.

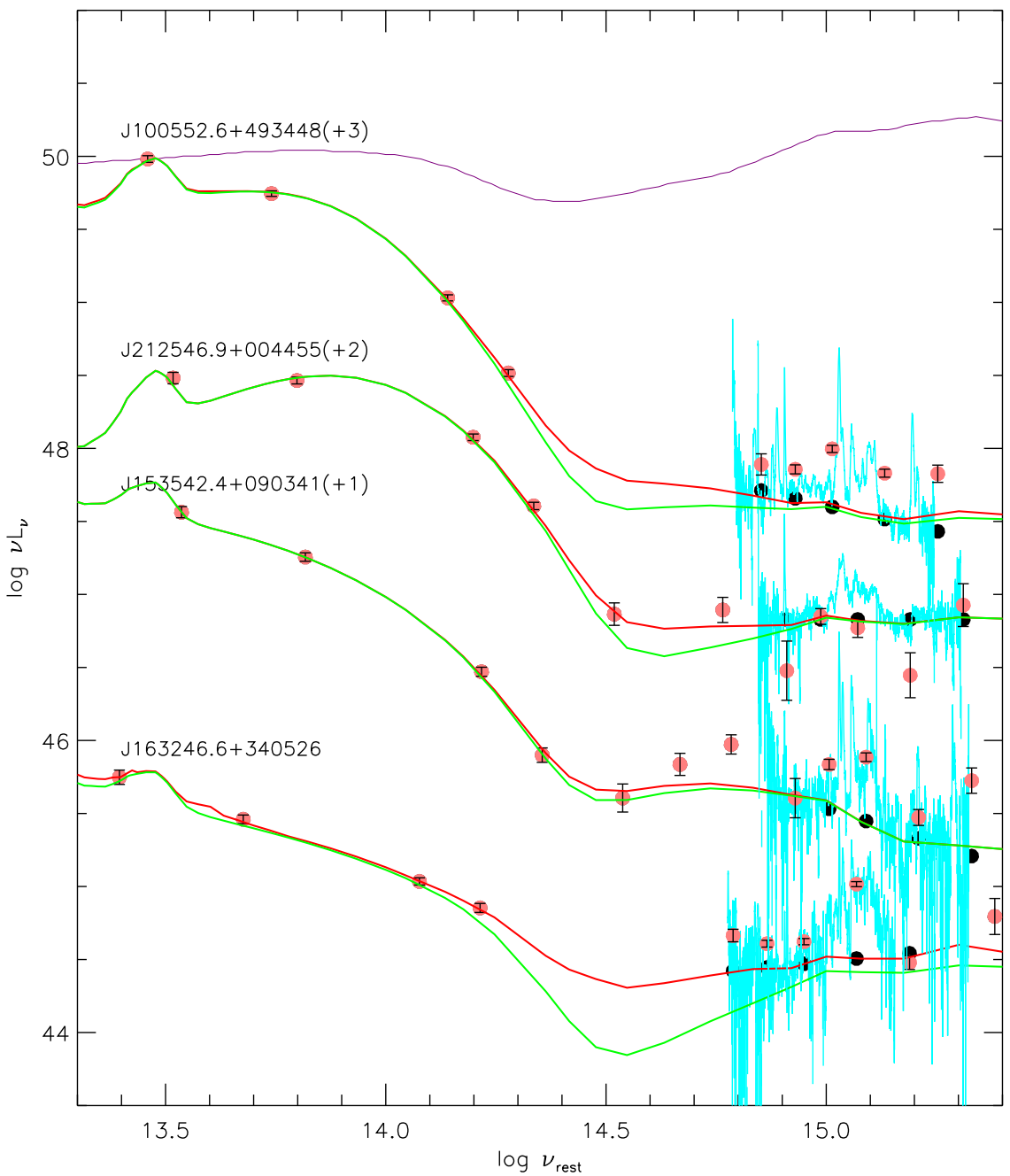

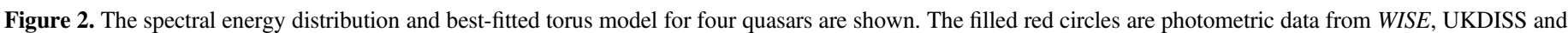

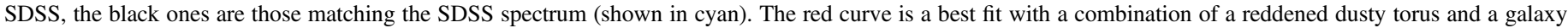

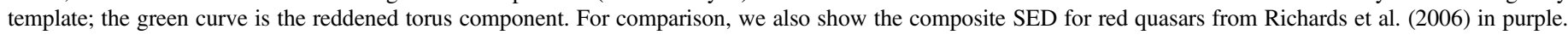

even lower and the optical and UV continuum from the dusty torus should be highly extinguished.

\section{DISCUSSION}

\subsection{On the Continuum Emission}

The large infrared to optical/UV ratio and the steep near to mid-infrared ratio indicates that the optical to UV, and even the near-infrared, emission from the accretion disk is obscured. Assuming the mid-infrared emission, which is dominated by the dusty torus emission as suggested by SED fitting, is nearly isotropic, the continuum emission at rest frame $3000 \AA$ is a factor of 30-300 weaker than in normal quasars (refer to Figure 1). If the $3000 \AA$ flux is attributed to the transmitted light, the dust extinction would be $E(B-V)=0.65-1.06$ for an SMC-like extinction curve. Applying such dust extinction to the average UV spectrum of quasars, we will get a slope $\beta=3.1-6.0\left(f_{\lambda} \propto \lambda^{+\beta}\right)$ between 2000 and $3000 \AA$, which is much steeper than the observed one. Adopting an average Galactic extinction curve will yield only slightly smaller slope. 
This indicates that the observed UV continuum is reflected/ scattered light. In fact, the residual ultraviolet flux can be well accounted for by reflected light from the clumpy dust torus itself as in the SED fitting. Alternatively, the continuum can be due to electron scattering, which is known to be important in Seyfert 2 galaxies (e.g., Antonucci \& Miller 1985). This suggests that the optical depth to the continuum emission region is much larger than indicated by the $E(B-V)$ derived above. In either case, the continuum should be polarized, and future polarization observation could verify this.

In the SED fit, we found that the dusty torus has a large subtending angle, i.e., covering $71 \%-87 \%$ of the sky. The large inclination angle suggests that our line of sight to the continuum source is blocked by the torus, consistent with weak optical and UV emission. In this case, the hot dust in the illuminated face of inner dust torus is also blocked, leading to a steep rise from the near to mid-infrared. So the dusty torus can naturally explain the obscuration and infrared SED. The SED fitting also suggests that the contribution of stellar light to the near and mid-infrared is small, which implies a high Eddington ratio.

Once the direct continuum is obscured, the large line equivalent width can be understood if the emission-line region is more extended than the dusty torus. In a few nearby Seyfert galaxies, the warm few hundred $\mathrm{K}$ dust which is responsible for mid-infrared emission is resolved into a thick structure of a few parsecs with a bolometric luminosity around $10^{44} \mathrm{erg} \mathrm{s}^{-1}$ (Tristram et al. 2007, 2014; López-Gonzaga et al. 2014). Note that this is not the outer radius of the torus, which might extend to several tens of parsecs. Since the size of the torus is proportional to $L^{1 / 2}$, with an infrared luminosity of $10^{46-47} \mathrm{erg} \mathrm{s}^{-1}$ for our quasars, the true size will be at least several tens of parsecs. In either case, the emission line region should be larger than this.

Before making a detailed discussion the size of the obscurer, let us consider the constraint on the size of emission line region first. The emission line width (FWHM), as fitted with $\mathrm{Mg}$ II, is in the range $900-8000 \mathrm{~km} \mathrm{~s}^{-1}$, which is broader than narrow lines in Seyfert galaxies, but is comparable with or narrower than the broad lines. Naively, this can be interpreted as lines that come from an intermediate region between the traditional BLR and NLR (Hu et al. 2008; Li et al. 2015). However, note that SDSS J1005+4934 also displays strong forbidden lines of similar width $\left(\sim 3000 \mathrm{~km} \mathrm{~s}^{-1}\right)$, different from traditional intermediate broad lines. Thus, we consider it more likely that these emission lines come from the inner region of NLR, and that the large width is due to outflows (e.g., Komossa et al. 2008; Zhang et al. 2011). In this picture the traditional BLR is completely obscured.

While the dusty torus provides the most naturally explanation for the obscuration, it is worth considering other alternatives. The obscurer can be a single dusty cloud in the clumpy torus model or the distant interstellar medium. The obscuration of the BLR requires a minimum size cloud to be $R_{\mathrm{BLR}} \simeq 0.1\left(\lambda L_{\lambda, 5100} / 10^{45} \mathrm{erg} \mathrm{s}^{-1}\right)^{0.5} \mathrm{pc}$ (Bentz et al. 2013). Using the infrared luminosity as an approximation for the bolometric luminosity and a bolometric correction to $L_{\mathrm{bol}} / \lambda L_{\lambda, 5100} \sim 9$, we estimate BLR sizes in the range of $0.1-0.3 \mathrm{pc}$. Assuming a Galactic dust to gas ratio $\left(N_{\mathrm{H}} / E(B-V)=(2.0-2.5) \times 10^{-22} \mathrm{~cm}^{2}\right.$, Bohlin et al. 1978; Cox et al. 2006), the gas column density of the obscurer should be much greater than $4 \times 10^{21} \mathrm{~cm}^{-2}$ as discussed above. Note that in the clumpy torus, the gas density should be of the order of a few $10^{6} \mathrm{~cm}^{-3}$ (Krolik \& Begelman 1988), so a BLR-sized cloud would have a column density two orders of magnitude larger than the above minimum value, or $E(B-V) \sim 100 \mathrm{mag}$ for a Galactic dust to gas ratio. On the other hand, assuming a gas density more typical of the interstellar medium $n_{\mathrm{H}} \sim 10^{2} \mathrm{~cm}^{-3}$, the minimum size of cloud would be several tens of parsecs to meet the minimum column density.

In the case of the single-cloud obscurer, one would expect to see long-term very large amplitude variability as the cloud moves in and out of the line of sight. The typical timescale would be the size of the cloud divided by the transverse speed, which is of order the local Keplerian velocity. In a typical galaxy, the orbit velocity is order of $500-1000 \mathrm{~km} \mathrm{~s}^{-1}$, depending on the distance to the center, and a minimum size of a cloud should be BLR sized, of order of $0.1-1 \mathrm{pc}$. This would give a variability timescale $\sim 10^{2-3}$ year. So it is difficult to observe the cloud transit event fully. However, the continuum source is much smaller (a hundred to thousand times smaller), so it is possible to observe transient obscuration of the continuum source with small changes in broad lines on timescales of years if the cloud has a sharp edge. There can also be cases where the cloud blocks the continuum source fully but only partially blocks the broad line region as the obscuring cloud moves in and out. These sources will show very large equivalent widths of broad lines. The probability of detecting such a source depends on the relative size of the obscuring cloud relative to the BLR size. In the case of comparable sizes of the two, one would expect that in most sources only the continuum is fully covered, while if the cloud is much larger than the BLR, most likely both continuum and BLR are obscured. Similarly, we will observe many more such partially obscured sources in the clumpy torus than in the smooth dust torus. In reality, the cloud may have a smooth edge so the contrast between emission line and continuum should be much moderate. We note that a single cloud occultation event is likely to give a smaller polarization degree than the dusty torus obscuration due to different symmetries (Marin et al. 2015).

The weakness of the optical to UV continuum and the strong mid-infrared emission may be due to a temporarily shut-off of the central engine. A comparison of infrared, optical, and UV fluxes requires a drop in the UV flux from the inner disk by a factor of 30-300 in these sources, compared to typical AGN (see Figure 2). The size of the mid-infrared emission region is of order of tens to hundreds of dust sublimation radii at the infrared luminosity according to our model of the SED (Hönig \& Kishimoto 2010). At the infrared luminosity of these sources, the light-crossing time of the mid-infrared emission region is of order ten to a hundred years, so the central engine would have to have shutdown in less than this time. A comparison of SDSS photometric data with Palomar Observatory Sky Survey on a timescale of 50 years in the observer frame shows a small fraction of quasars with a variability amplitude of $3 \mathrm{mag}$ in $g$-band (de Vries et al. 2005). Although these amplitudes are still smaller than that is required, since we do not have light curves of AGN on such long timescales, it is impossible to tell whether such a large amplitude variability happened on timescale of a hundred years and how frequent they are. Thus this remains a possibility.

There are several tests that could distinguish among these possibilities. First, scattered light should be highly polarized, while direct light from a much less active accretion disk is 
likely not. The observed scattered light in Seyfert 2 galaxies is polarized by a few to tens' percent, while the polarization of direct emission from quasars is usually less than 1\% (Marin et al. 2015). It should be noted that if a quasar resides in the gas-rich environment, there is inevitable scattered light from surrounding gas. As the central continuum source dims quickly, the fraction of scattered light increases, and so does the polarization degree. So a more critical test would be to observe the transmitted hard X-rays. Second, direct emission from accretion disk is likely to be variable on relatively short timescales, while the scattered light is not. Thus, the detection of variability on timescales of less than a year would support the intrinsic weak continuum emission model.

\subsection{The Origin of Strong Fe II Emission}

We now turn to the properties of the emission region. Previous studies suggested that quasar emission lines consist of distinct kinematic components: broad, intermediate and narrow components (Brotherton et al. 1994; Marziani et al. 1996). Hu et al. (2008) found that optical Fe II lines are formed in the intermediate region, that is redshifted with respective to the system.

The Fe II spectrum of our sources does not look like those in normal quasars or from most theoretical simulations aimed at reproducing observed Fe II in I Zw 1 (Sigut \& Pradhan 2003; Baldwin et al. 2004; Bruhweiler \& Verner 2008; Ferland et al. 2009). We will mainly focus on the Fe II emission in SDSS $\mathrm{J} 1535+0903$ first, because in other sources the spectra can be reproduced with a combination of SDSS J1535+0903 and I Zw 1-like components. The UV Fe II emission is dominated by a few bumps, namely multiplets UV 1, UV 2,3, UV 62,63, with weak emission in between and very weak or no emission at wavelengths longer than $2900 \AA$. In SDSS J1005+4934, the SDSS spectrum extends to $4400 \AA$ in the quasar rest frame, and optical Fe II lines appear relatively weak.

For thermal excitation, the extreme spike/gap ratio of 8.5 in SDSS J1535+0903 may indicate very high densities $\left(n_{\mathrm{H}}>10^{13} \mathrm{~cm}^{-3}\right)$ and very low ionization parameters $\left(U=10^{-4-5}\right.$ ), as shown in Figure 3 of Baldwin et al. (2004). But their models are only for the broad line region, and the ionizing photon flux is too large for our case. However, in their figure, the curves of iso-spike/gap ratio run diagonally, so the dimensionless ionization parameter may be more crucial than the density. Unfortunately their models do not extend to the low density and very low ionization regime, so it is not clear whether extreme spike/gap ratios can be reproduced there. But if this is indeed the case, the Fe II emission region must be far from the quasar so that it is not obscured by the dusty torus. At very low values of the ionization parameter, some other lines, such as $\mathrm{C}$ II and Ni II, might become strong. Detailed photoionization modeling would be required. Also the presence of strong $\mathrm{C}_{\text {IV }}$ and $\mathrm{Al}$ III , and the lack of $\mathrm{C}$ III and $\mathrm{Si}$ III cannot be explained by this model.

Alternatively, the extended emission may come from resonant scattering in low-ionization quasar outflows. Previous observations suggest that FeLoBALs and HiBALs, at least in some quasars, are formed on the scale of $100 \mathrm{pc}$ to a few $\mathrm{kpc}$ (see references in Section 1), so it is likely that outflows are not obscured by the putative dusty torus that blocks the line of sight to the central continuum in Type 2 quasars. The outflows may produce Fe II via collisional excitation, as well as via continuum fluorescence. The contribution of fluorescence
Table 2

Torus Models for Spectral Energy Distribution

\begin{tabular}{lccccc}
\hline \hline SDSS J & $f_{\text {gal }}$ & $\begin{array}{c}\text { Inclination } \\
\text { (degree) }\end{array}$ & $\begin{array}{c}\Theta_{0} \\
(\text { degree })\end{array}$ & $\alpha$ & $\tau_{\mathrm{cl}}$ \\
\hline $100552.6+493448$ & 0.048 & 15 & 60 & -1.5 & 30 \\
$153542.4+090341$ & 0.003 & 15 & 45 & -0.5 & 80 \\
$163246.6+340526$ & 0.086 & 35 & 60 & -1.0 & 80 \\
$212546.9+004455$ & 0.005 & 15 & 60 & -2 & 50 \\
\hline
\end{tabular}

Note.

a Columns are (2) fraction of galaxy light in $2-10 \mu \mathrm{m}$ band, (3) the angle between the line of sight and the equator of torus; (4) the half subtending angle of torus; (5) the power-law index of cloud distribution as a function of distance to the AGN; (6) the optical depth of individual cloud in $V$.

relative to collisional excitation depends on the optical depth of Fe II as well as the density and temperature of the gas. In the case of FeLoBAL outflows, the density is usually low (order of $10^{3-5} \mathrm{~cm}^{-3}$, de Kool et al. 2001; Lucy et al. 2014; c.f., Zhang et al. 2015), and the optical depth of most Fe II lines is usually not large (order of a few to tens for strong lines). Thus, continuum fluorescence may be the dominant process in the outflows for Fe II lines.

Some other emission lines may be also dominated by resonant scattering from such outflows. Consequently, these lines should appear strong in comparison with non-resonant lines. The presence of strong $\mathrm{Al}$ III , but very weak or missing similar component of $\mathrm{C}$ III] and $\mathrm{Si}$ III], which are usually much stronger, in SDSS J1535+0903, indicates that resonant scattering is the dominant excitation mechanism in this object. Al III can be produced by either resonant scattering or collisional excitation, while $\mathrm{C}$ III] and $\mathrm{Si}$ III are only collisionally excited. Although the relative strength of $\mathrm{Al}$ III increases with gas metallicity, this does not explain the fact that $\mathrm{Al} \mathrm{III} \mathrm{is} \mathrm{much}$ stronger than $\mathrm{C}$ III]. In Table 2 , the $\mathrm{Al}$ III equivalent width is a factor of 3.7 larger than the $\mathrm{C}$ III] equivalent width. As discussed in Section 3.1, the observed UV continuum is likely scattered light, and, as seen in Figure 1, the equivalent widths of $\mathrm{C}$ III] and $\mathrm{Si}$ III is similar to that of I ZW 1. So C III] emission may be fully accounted for by scattered component and the contribution from the outflow component may be negligible.

If $\mathrm{Fe}$ II is produced by continuum fluorescence in outflows, a simple idea is that its spectrum may resemble those of the Fe II absorption line spectra in FeLoBAL QSO SDSS $\mathrm{J} 080957.39+181804.42$. We note that there are usually very weak Fe II absorption lines above $2800 \AA$, and no optical Fe II absorption lines, consistent with the observed Fe II in these spectra. To test this idea, we extract the absorption line spectrum of a FeLoBAL QSO. This spectrum is shown in Figure 1, and is similar, to first order. It should be noted that the spectrum of fluorescent emission is not exactly the same as the Fe II absorption spectrum, because an $\mathrm{Fe}_{\text {II }}$ ion in an excited level, after absorbing a photon, may decay to a different lower level, thus emitting a photon with an energy different from the absorbed one, especially when resonance lines become optically thick. Detailed calculations are presented in Appendix.

In Appendix, we demonstrate that a pure fluorescent model can indeed reproduce the observed Fe II band ratios in SDSS $\mathrm{J} 1535+0903$ very well. In order to make Fe II the dominant ion of $\mathrm{Fe}$, we let the incident ionizing continuum be filtered by an intervening absorbing gas of column density $10^{23} \mathrm{~cm}^{-2}$. We 


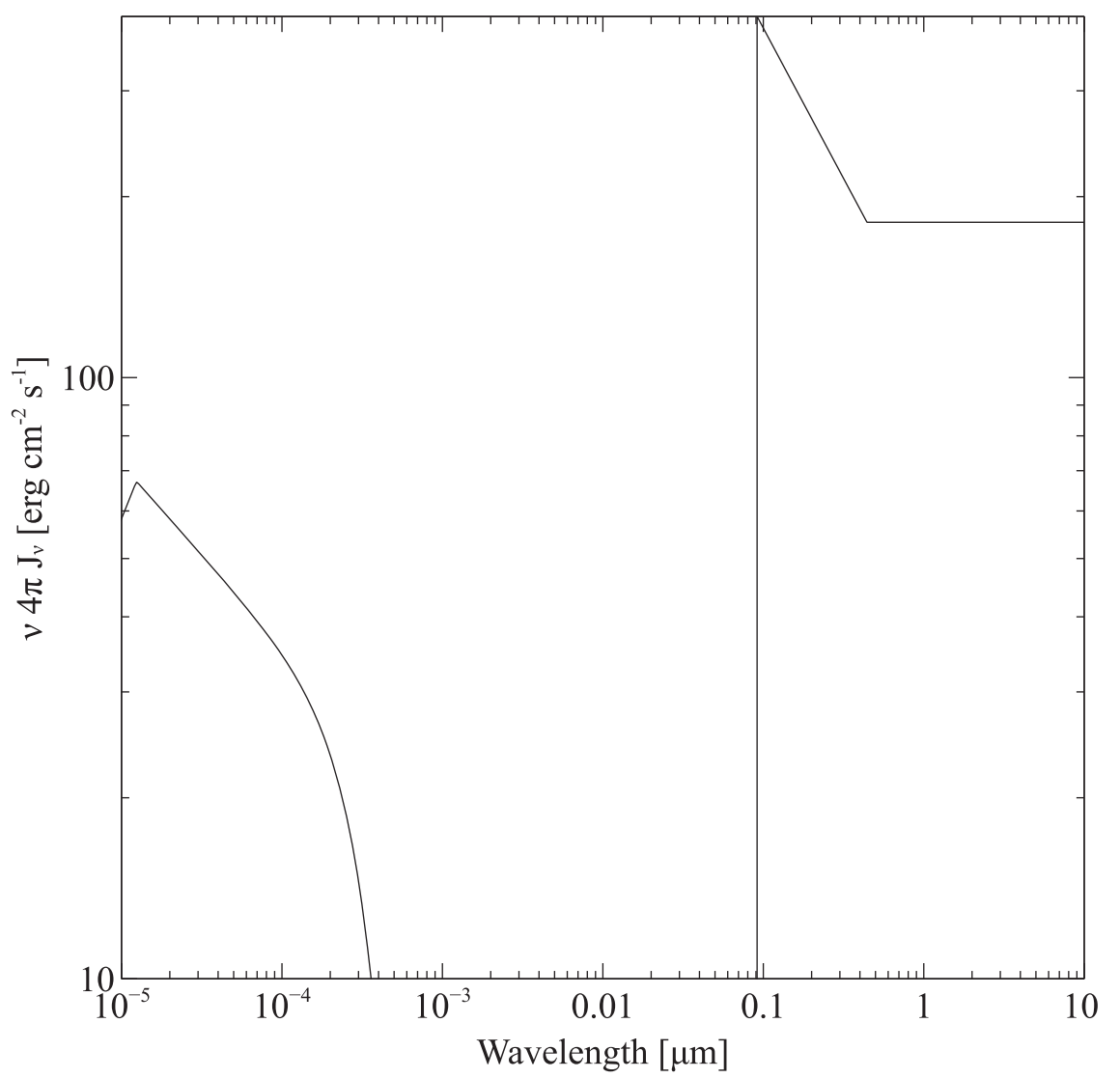

Figure 3. The SED striking the outer cloud. It is a simple AGN SED extinguished by a column density $N(\mathrm{H})=10^{23} \mathrm{~cm}^{-2}$, which removes light shortward of the Lyman limit. The conditions in the cloud are mainly affected by the Balmer continuum.

adopt a gas density $n_{\mathrm{H}}=10^{4.5} \mathrm{~cm}^{-3}$, consistent with those in the FeLoBAL QSOs, and a turbulent velocity of $1000 \mathrm{~km} \mathrm{~s}^{-1}$ to mimic the outflow velocity. We find that at the optical depth of the resonant $\mathrm{Fe}$ II line at $\tau(2599 A)$ is 900 , and that the model produces UV FeII band ratios that are consistent with the observed ones. As far as we know, this is the only model that gives such an extreme $\mathrm{Fe}$ II ratio at a low ionizing continuum flux, which is consistent with its large line formation region. ${ }^{5}$ Furthermore, the model gives weak Fe II lines at wavelength longer than $2800 \AA$, which is also consistent with the observation. The best matched model predicts an equivalent width of Fe II at $2355 \AA$ band of $99 \AA$, which is about one 6th of the observed value for SDSS J1535+0903, for a covering factor of one. However, as we discussed in Section 3.1, the continuum incident to the gas is obscured and the observed continuum is attenuated by two orders of magnitude, so only a few percentages covering factor would be sufficiently to explain the observed Fe II strength. This covering factor is consistent with the estimate in next subsection. The gas producing the Fe II emission is cool, as shown in the Appendix, so collisional excitation is unimportant. This accounts for the weak optical Fe II emission.

Note that this type of model will also predict a number of other low ionization lines, such as $\mathrm{Ti}$ II, Ni II and $\mathrm{Si}$ II, but their atomic data are very sparse. So a full treatment of these lines has to wait for a major update in the atomic data. Our models in Appendix do not produce $\mathrm{Al}$ III and $\mathrm{C}$ IV, because ionizing

\footnotetext{
5 Some models in Baldwin et al. (2004) also show such extreme line ratios but at high ionizing photon flux.
}

photons above $13.6 \mathrm{eV}$ are required to produce these ions while in our scenario these photons are intentionally filtered out to prevent destruction of $\mathrm{Fe}^{+}$ions. Both $\mathrm{Al}$ III and $\mathrm{C}$ IV are observed in absorption in a FeLoBAL QSO, and their average emission spectrum should not be different from the absorption line spectrum in a FeLoBAL QSO because they are scattered resonantly. So our purpose is only to demonstrate the effect of $\mathrm{Fe}$ II radiation transfer after $\mathrm{Fe}$ II resonant absorption.

Note also that the observed $\mathrm{Fe}$ II ratios from different $\mathrm{Fe}$ II bands depends on the reddening, because FeloBAL QSOs often show a large internal dust extinction. We do not apply such a reddening correction because we do not know the location of the dust. If dust lies outside of the BALR, then an extinction correction should be applied. On the other hand, if dust is within the BALR, no correction is needed. Anyway, to first order the absorbed line spectrum of FeLoBAL quasars are similar to the quasars observed in the sample.

In SDSS J1005+4934, we also detect strong high ionization forbidden or semi-forbidden lines that are excited by collisional process, while the $\mathrm{Fe}$ II spectrum as well as $\mathrm{Fe}$ II $/ \mathrm{Al}$ III ratio, are nearly the same as SDSS J1535+0903. We also obtained an electron density of $(3-4) \times 10^{5} \mathrm{~cm}^{-3}$ from $\mathrm{C}_{\text {III }}$ and Si III. These lines are rather broad with widths of $3000 \mathrm{~km} \mathrm{~s}^{-1}$. The presence of such lines does not necessarily contradict our interpretation of the strong $\mathrm{Fe}$ II, $\mathrm{Al}$ III and $\mathrm{Mg}$ II lines as resonantly scattering from an outflow. According the the recent model of Faucher-Giguère et al. (2012), the FeLoBAL may be produced in the shocked cooling gas during interaction of outflows with the cold gas. The gas producing strong high ionization forbidden lines may be the warm phase of such 


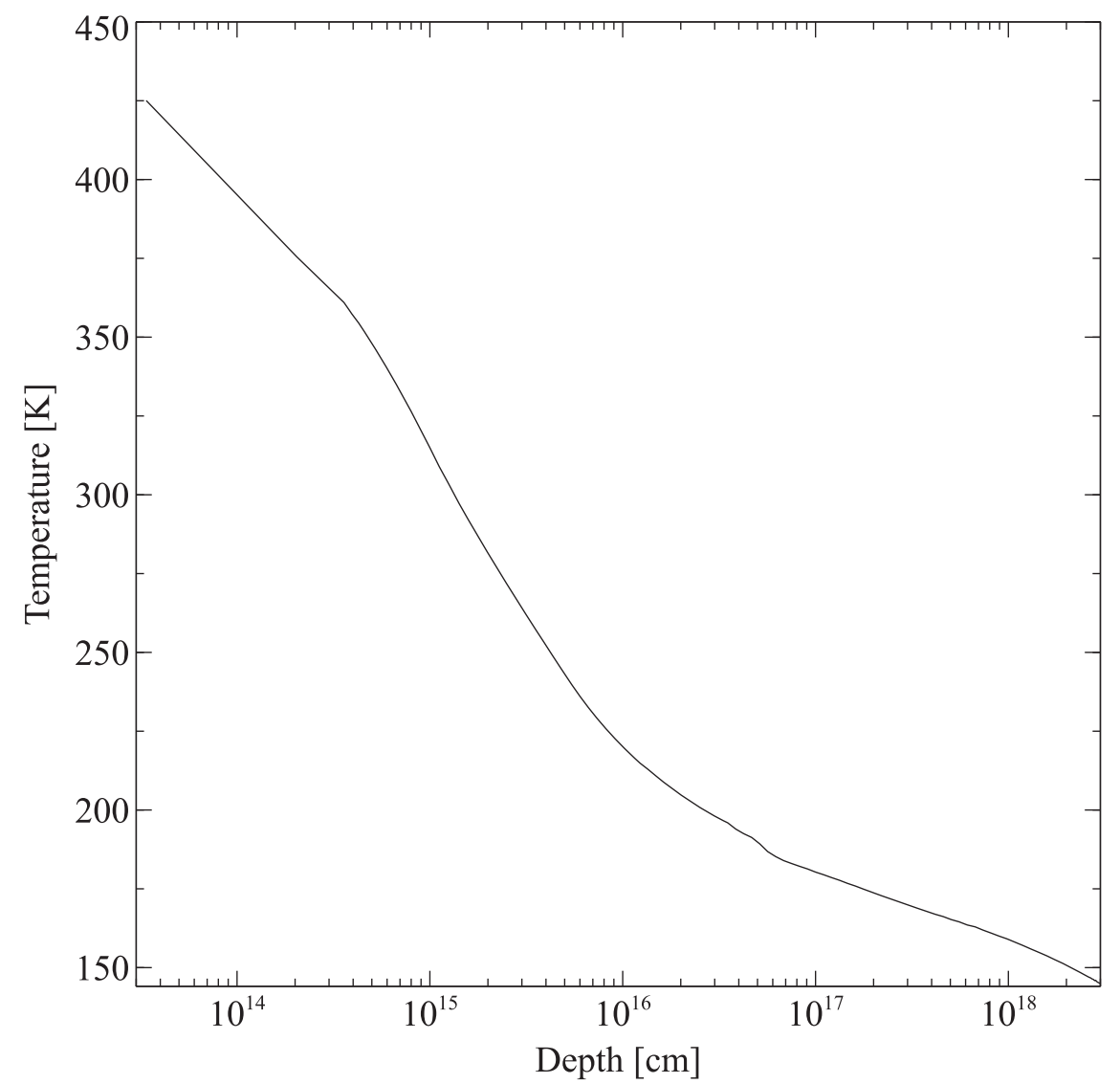

Figure 4. The computed temperature structure. The vertical axis gives the temperature $[\mathrm{K}]$ while the horizontal axis gives the depth into the cloud in $\mathrm{cm}$. The illuminated face of the cloud reaches temperatures a bit over $400 \mathrm{~K}$ while the shielded face is much cooler, approaching $100 \mathrm{~K}$. These temperatures are all too cool to permit collisional excitation of optical or UV Fe II lines.

shocked gas, and the large width of the line may be explain in this way as well.

Blue shifted Fe II and Mg II lines are seen in the SDSS J1632 +3405 , while emission lines in other quasars do not display systematic blueshift. The blueshifted lines can be easily explained as from an outflow since one expects that the far side of the outflow is subject to large dust extinction and would not be seen. However, emission lines from outflows are not necessarily systematically blueshifted if the farside of the outflows is not particularly obscured or the system is seen nearly edge-on. In the continuum fit, we found that SDSS $\mathrm{J} 1632+3405$ has the smallest inclination angle among these objects.

\subsection{Constraints on the Covering Factor for the Scattered Region}

If the $\mathrm{Fe}$ II in these objects is due to fluorescent excitation, we can constrain the global covering factor of the outflows in these objects. To first order, we can assume an isotropic angular redistribution function, so the scattered $\mathrm{Fe}$ II flux will be

$$
f_{\mathrm{sc}}(\lambda)=\left[1 .-\exp \left(-\tau_{\lambda}\right)\right] f_{c}(\lambda) * C
$$

where $f_{c}$ is the un-absorbed continuum flux. Assuming a maximum $\tau>1$ at certain $\lambda$, one can then estimate a minimum covering factor $C \simeq\left[f_{\mathrm{sc}} / f_{c}(\lambda)\right]_{\max }$. To estimate $f_{c}$, we assume that the intrinsic SED is similar to the mean quasar SED, and scale the latter to match the WISE luminosity at $22 \mu \mathrm{m}$, the peak wavelength of SED. By doing so, we obtain covering factors of $0.05,0.20,0.01$, and 0.06 for SDSS J2125+0044, SDSS J1632+3405, SDSS J1005+4934 and J1535+0903. Note that if $\mathrm{Mg}$ II is dominated by resonant scattering in the BALR, then the covering factor of $\mathrm{Mg}$ II BALR would be a factor of 2-3 times larger than the above value. There are several uncertainties in this estimate.

First, due to large number of energy levels, Fe II cannot be rigidly considered as resonant scattering, but rather as the fluorescent process. The absorbed photons may emerge at other wavelength, so the strong resonant lines are usually weaker than in the case of resonant scattering. Second, it is possible that infrared emission of these quasars is enhanced because we selected objects with very red infrared colors. In this case, the unabsorbed continuum is over-estimated. Since SED modeling suggested that infrared emission is dominated by the AGN component, while it was shown that, on average, thermal dust emission accounts for $35 \%$ of the total power of quasars (e.g., Ma \& Wang 2013), thus at most, the intrinsic continuum is over-estimated by a factor of $2-3$. Third, the scattered photons may be destroyed by dust within or outside the BALR, so we may underestimate the covering factor. It has been known for quite a long time that FeLoBAL QSOs are reddened. But the location of the dust is still unknown. Recently, there are arguments that dust may be mixed within the BALR because near-infrared emission is correlated with the outflow velocity (Zhang et al. 2014), and the variability of BAL is correlated with the UV spectral slope (He et al. 2015). Further, Dunn et al. (2015) showed that reddening must be caused by large scale 


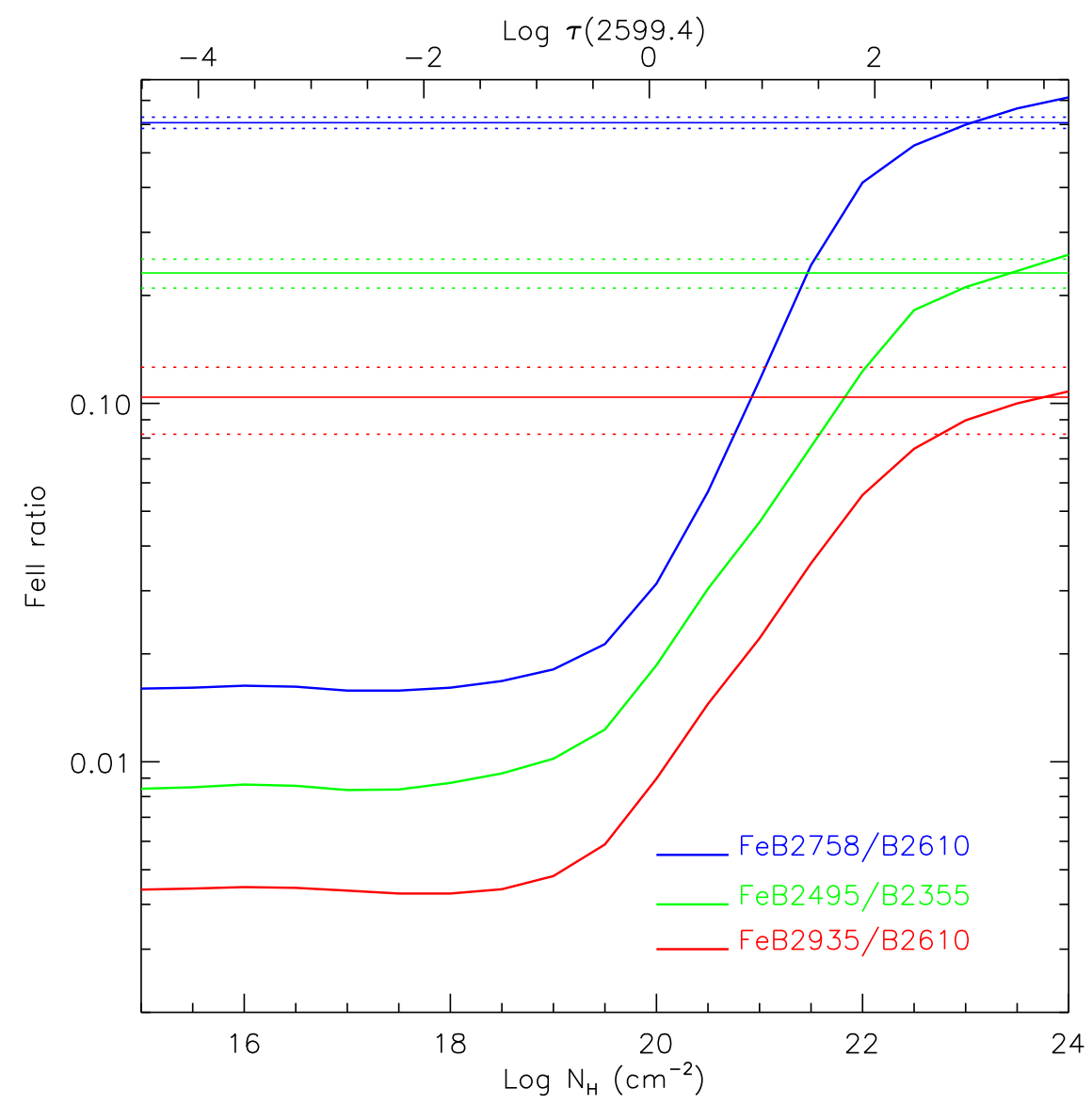

Figure 5. Band ratios as a function of total hydrogen column density on the bottom and the optical depth in a strongly absorbing UV line on the top. The horizontal bar indicates the observed band ratio (solid) and its $68 \%$ uncertainties (dashed).

dust because both the continuum and narrow lines are reddened. If dust is outside of the BALR and the scattered light suffers the same extinction as the continuum source, and adopting a median value for the continuum extinction for LoBAL $E(B-V)=0.05$ (Dunn et al. 2015), the correction will raise the covering factor by about $40 \%$. Despite these uncertainties, we note that in 3 of 4 objects, the uncorrected covering factors are already larger than 0.05 , while according to statistics, FeLoBAL QSOs account for no more than $2 \%$ of total quasars (Trump et al. 2006; Dai et al. 2012). This suggests strongly that FeLoBAL QSOs must be in a special phase of quasar evolution, rather than representing a very small covering factor.

\section{CONCLUSIONS}

We presented an analysis of four quasars with peculiar UV Fe II spectra. They had extremely strong UV1, 3, 4, 62, 63 and very weak Fe II at wavelengths longer than 2800. Their SEDs rise very steeply from the optical to the mid-infrared, suggesting they are obscured. The obscurer is most likely the dusty torus, since that also provides a self-consistent explanation for the infrared emission although other alternatives cannot be ruled out. The characteristics of the Fe II spectra can be understood as due to resonant scattering in an extended outflow since the size of the scattering region must be outside the obscuring torus and its Fe II spectrum similar to absorption spectrum of FeLoBALs. Our fluorescent models can successfully produce the observed $\mathrm{UV} \mathrm{Fe}_{\mathrm{II}}$ band ratios and the weakness of $\mathrm{Fe}$ II lines at wavelengths longer than $2800 \AA$ with a low ionizing photon fluxes. This is because the strong UV lines are excited by continuum pumping within gas that is too cool for collisional excitation to be significant. Strong Al III and weak $\mathrm{C}_{\text {III] }}$ and $\mathrm{Si}$ III emission may be explained as resonant scattering, as is observed in FeLoBAL QSOs. The obscuration of the direct continuum also explains the very large equivalent widths of $\mathrm{Fe}$ II, $\mathrm{Mg}$ II and $\mathrm{Al}$ III lines. These objects are misaligned FeLoBALs. By considering the amount of scattered light we set a lower limit on the covering factor of FeLoBALs to be $5 \%-20 \%$ in three objects, supporting the scenarios that FeLoBAL QSOs are a special stage of quasar evolution.

We thank the referee for constructive comments that help to improve the presentation of the paper. We acknowledge the financial support by the Strategic Priority Research Program "The Emergence of Cosmological Structures" of the Chinese Academy of Sciences (XDB09000000), NSFC (NSFC11233002, NSFC-11421303, U1431229) and National Basic Research Program of China (grant No. 2015CB857005). Funding for SDSS-III has been provided by the Alfred P. Sloan Foundation, the Participating Institutions, the National Science Foundation, and the U S Department of Energy Office of Science. The SDSS-III web site is http://www.sdss3.org/. SDSS-III is managed by the Astrophysical Research Consortium for the Participating Institutions of the SDSS-III Collaboration. GJF acknowledges support by NSF (1108928, 1109061, and 1412155), NASA (10-ATP10-0053, 10ADAP10-0073, NNX12AH73G, and ATP13-0153), and STScI 


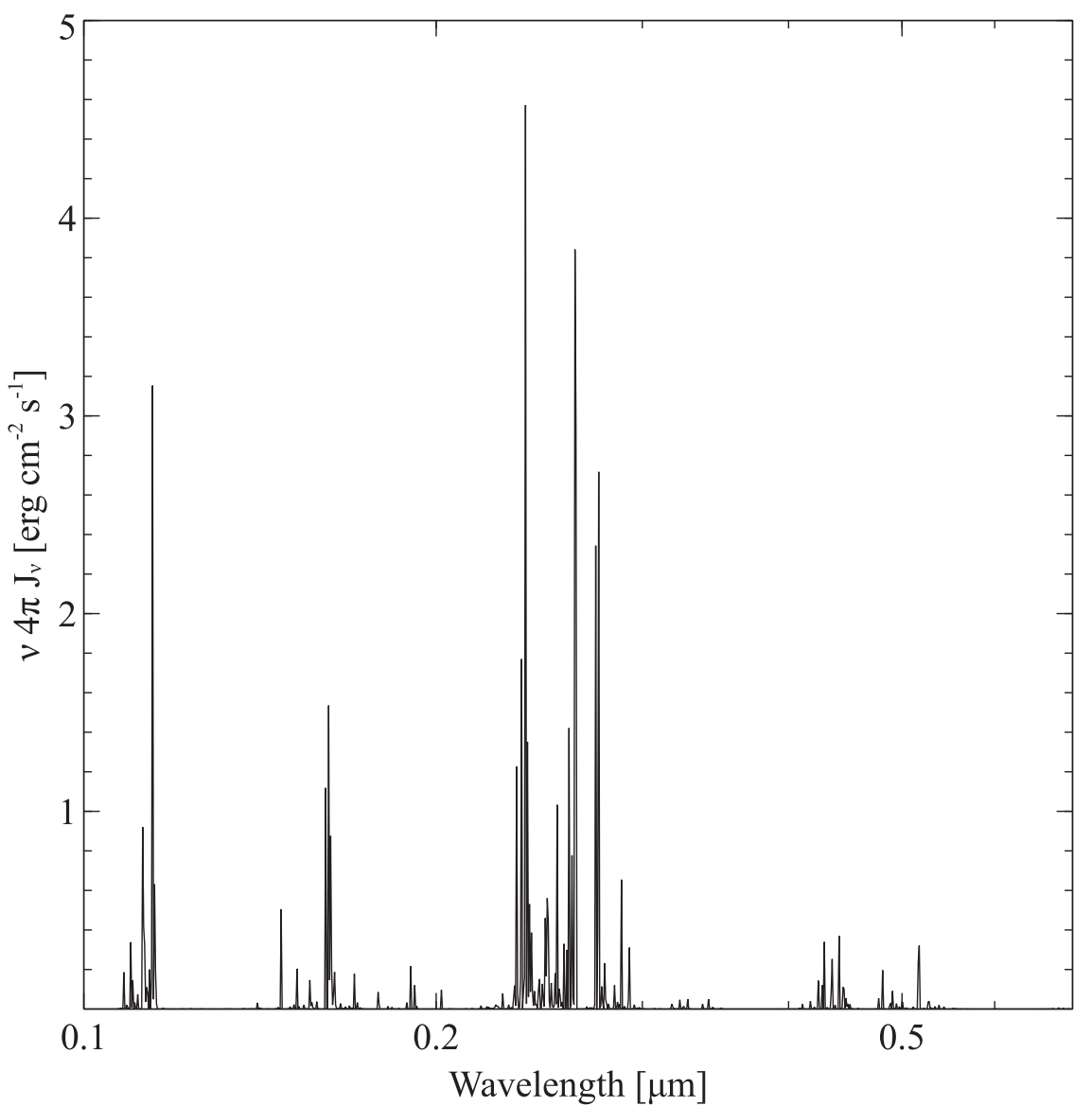

Figure 6. The net Fe II emission from the cloud. The horizontal axis gives the wavelength in microns and the vertical axis gives the emission produced by the cloud. The incident continuum is not included since we assume that it is not directly visible to us.

(HST-AR- 13245, GO-12560, HST-GO-12309, GO13310.002-A, HST-AR-13914, and HST-AR-14286.001).

\section{APPENDIX \\ FLUORESCENT Fe II EMISSION}

Here we examine the properties of a cold cloud where iron is mainly singly ionized. Fe II emission will be produced by continuum fluorescence under these circumstances. Our scenario is that an intervening cloud has extinguished the AGN continuum and that this attenuated SED strikes an outer dust-free cloud. The intervening cloud is assumed to have a column density of $N(\mathrm{H})=10^{23} \mathrm{~cm}^{-2}$ and an unattenuated ionization parameter of $\log U=-1.5$ (although this has little affect on our predictions). The SED transmitted through this cloud, which then strikes the outer cloud, is shown in Figure 3.

We assume that the outer cloud is dust free. This is to allow Fe to exist in the gas phase. Fe is strongly depleted in dusty gas (Jenkins 2009) so little $\mathrm{Fe}$ II emission occurs when grains are present. It is possible that the cloud was at one time too close to the AGN for dust to exist.

The cloud would be similar to a classical PDR (Röllig et al. 2007) were grains present. In a PDR grains heat the gas while absorbing the Balmer continuum of the incident SED. Since grains are, by hypothesis, absent in our cloud, the gas will be heated mainly by photoelectric absorption by the gas, mainly of second and third row elements with ionization potentials smaller than hydrogen. A low gas kinetic temperature results. The radiation striking the gas is bright at wavelengths longward of the Lyman limit, so the main species that will be present in the cloud will be $\mathrm{H}_{\mathrm{I}}, \mathrm{He}_{\mathrm{I}}, \mathrm{C}_{\mathrm{II}}, \mathrm{N}_{\mathrm{I}}, \mathrm{O}_{\mathrm{I}}$, $\mathrm{Ne}_{\mathrm{I}}, \mathrm{Na}$ II, $\mathrm{Mg}$ II, $\mathrm{Al}_{\text {III, }} \mathrm{Si}_{\text {II, }}, \mathrm{S}$ II, and Fe II. Typical gas temperatures in our resulting models are around $300 \mathrm{~K}$ (Figure 4), too cool to collisionally excite optical or UV lines. UV line emission is produced by continuum fluorescent excitation in this case. Note that it is quite likely that the SED striking the cloud is not visible from our line of sight. This would occur if parts of the torus lie between us and the central object.

We use version C13.03 of Cloudy, a spectral simulation code last described by Ferland et al. (2013). Verner et al. (1999) describe the Fe II model in this version of Cloudy. Fe II emission is complex (Baldwin et al. 2004) and we report it using the sums over wavelength bands described in that paper.

Results are mainly sensitive to the column density of the outer cloud and we will show predictions resulting from varying this parameter below. In this cool environment, $\mathrm{Fe}_{\mathrm{II}}$ is excited mainly by absorption of the incident SED by UV lines whose lower levels are close to, or within, the ground term. The level populations within the ground term are affected by the density which then affects which UV lines are efficiently pumped. The density does not affect the emission from high levels so long as it is below the critical density of the excited levels, $\sim 10^{10}-10^{14} \mathrm{~cm}^{-3}$. At lower densities the emission spectrum is mainly determined by the branching ratios as electrons decay to lower levels. We experimented with a range of densities but settled on $\log N(\mathrm{H})=4.5 \mathrm{~cm}^{-3}$. 
Lines absorb mainly over their Doppler core so the amount of pumping will depend on the line width (Ferland et al. 1992). We assume $1000 \mathrm{~km} \mathrm{~s}^{-1}$ in the examples we show although this does not greatly affect the details. The Fe II grows stronger as the column density increases and $\mathrm{Fe}$ II lines absorb more of the incident continuum. Optical depths in the strong UV lines do affect the branching ratios. As they grow more opaque decays from their upper levels will favor subordinate lines. This is quite analogous to the transition from Case A to Case B in $\mathrm{H}$ I emission (Osterbrock \& Ferland 2006). Finally the intensity of the radiation field give in Figure 3 must be set. We assume an unabsorbed ionization parameter of $\log U=-1.7$.

Results are shown in Figure 5. The lower axis gives the total hydrogen column density. An iron abundances of $\log \mathrm{Fe} /$ $\mathrm{H}=-4.55$ was assumed. A good fit is obtained at $\log N(\mathrm{H})=23 \mathrm{~cm}^{-2}$. The upper axis shows the optical depth in one of the strong UV lines that is responsible for pumping the atom. The net Fe II emission is shown in Figure 6. Fe II UV $1,3,4,63,64$ are the strongest lines, while those at wavelengths longer than $2800 \AA$ are much weaker. The spectrum looks similar to the observed $\mathrm{Fe}$ II.

With these parameters, the thickness of the cloud is one parsec, and the distance to the continuum source is estimated about $1 \mathrm{kpc}$, following the definition of the ionization parameter, and the inferred intrinsic continuum luminosity of $\mathrm{J} 1535+0903$. The total volume of $\mathrm{Fe}$ II region is about $10^{6} \mathrm{pc}^{3}$, and a total gas mass of about $10^{9} M_{\odot}$, which is comparable to the reported $10^{8-9} M_{\odot}$ molecular gas in outflows of infrared luminous quasars, such as Mrk 231 (Feruglio et al. 2010; Cicone et al. 2014). However, the gas density is poorly constrained so these numbers are only very rough estimate.

\section{REFERENCES}

Antonucci, R. R. J., \& Miller, J. S. 1985, ApJ, 297, 621

Baldwin, J. A., Ferland, G. J., Korista, K. T., Hamann, F., \& LaCluyzé, A. 2004, ApJ, 615, 610

Baskin, A., Laor, A., \& Hamann, F. 2013, MNRAS, 432, 1525

Bentz, M. C., Denney, K. D., Grier, C. J., et al. 2013, ApJ, 767, 149

Bohlin, R. C., Savage, B. D., \& Drake, J. F. 1978, ApJ, 224, 132

Boksenberg, A., Carswell, R. F., Smith, M. G., \& Whelan, J. A. J. 1978, MNRAS, 184, 773

Borguet, B. C. J., Arav, N., Edmonds, D., Chamberlain, C., \& Benn, C. 2013, ApJ, 762, 49

Boroson, T. A., \& Meyers, K. A. 1992, ApJ, 397, 442

Brotherton, M. S., Wills, B. J., Francis, P. J., \& Steidel, C. C. 1994, ApJ, 430, 5

Bruhweiler, F., \& Verner, E. 2008, ApJ, 675, 83

Chamberlain, C., Arav, N., \& Benn, C. 2015, MNRAS, 450, 1085

Cicone, C., Maiolino, R., Sturm, E., et al. 2014, A\&A, 562, A21

Cox, N. L. J., Cordiner, M. A., Cami, J., et al. 2006, A\&A, 447, 991

Dai, X., Shankar, F., \& Sivakoff, G. R. 2012, ApJ, 757, 180

de Kool, M., Arav, N., Becker, R. H., et al. 2001, ApJ, 548, 609

de Vries, W. H., Becker, R. H., White, R. L., \& Loomis, C. 2005, AJ, 129,615

Dunn, J. P., Arav, N., Aoki, K., et al. 2012, ApJ, 750, 143
Dunn, J. P., Wasik, B., Holtzclaw, C. L., et al. 2015, ApJ, 808, 94

Fabian, A. C. 2012, ARA\&A, 50, 455

Farrah, D., Urrutia, T., Lacy, M., et al. 2010, ApJ, 717, 868

Faucher-Giguère, C.-A., Quataert, E., \& Murray, N. 2012, MNRAS, 420, 1347

Ferland, G. J., Hu, C., Wang, J.-M., et al. 2009, ApJL, 707, L82

Ferland, G. J., Peterson, B. M., Horne, K., Welsh, W. F., \& Nahar, S. N. 1992, ApJ, 387, 95

Ferland, G. J., Porter, R. L., van Hoof, P. A. M., et al. 2013, RMxAA, 49, 137

Feruglio, C., Maiolino, R., Piconcelli, E., et al. 2010, A\&A, 518, L155

Hall, P. B., Anderson, S. F., Strauss, M. A., et al. 2002, ApJS, 141, 267

Hamann, F., Korista, K. T., \& Morris, S. L. 1993, ApJ, 415, 541

He, Z.-C., Bian, W.-H., Jiang, X.-L., \& Wang, Y.-F. 2015, MNRAS, 454, 3962

Hönig, S. F., \& Kishimoto, M. 2010, A\&A, 523, A27

Hu, C., Wang, J.-M., Ho, L. C., et al. 2008, ApJL, 683, L115

Jenkins, E. B. 2009, ApJ, 700, 1299

Knigge, C., Scaringi, S., Goad, M. R., \& Cottis, C. E. 2008, MNRAS, 386, 1426

Komossa, S., Xu, D., Zhou, H., Storchi-Bergmann, T., \& Binette, L. 2008, ApJ, 680, 926

Korista, K., Bautista, M. A., Arav, N., et al. 2008, ApJ, 688, 108

Krolik, J. H., \& Begelman, M. C. 1988, ApJ, 329, 702

Laor, A., Jannuzi, B. T., Green, R. F., \& Boroson, T. A. 1997, ApJ, 489, 656

Lazarova, M. S., Canalizo, G., Lacy, M., \& Sajina, A. 2012, ApJ, 755, 29

Li, Z., Zhou, H., Hao, L., et al. 2015, ApJ, 812, 99

Liu, G., Arav, N., \& Rupke, D. S. N. 2015, ApJS, 221, 9

López-Gonzaga, N., Jaffe, W., Burtscher, L., Tristram, K. R. W., \& Meisenheimer, K. 2014, A\&A, 565, A71

Lucy, A. B., Leighly, K. M., Terndrup, D. M., Dietrich, M., \& Gallagher, S. C. 2014, ApJ, 783, 58

Ma, X., \& Wang, T. G. 2013, MNRAS, 430, 3445

Marin, F., Goosmann, R. W., \& Gaskell, C. M. 2015, A\&A, 577, A66

Marziani, P., Sulentic, J. W., Dultzin-Hacyan, D., Calvani, M., \& Moles, M. 1996, ApJS, 104, 37

Meusinger, H., Schalldach, P., Scholz, R.-D., et al. 2012, A\&A, 541, A77

Nestor, D., Hamann, F., \& Rodriguez Hidalgo, P. 2008, MNRAS, 386, 2055

Osterbrock, D. E., \& Ferland, G. J. (ed.) 2006, Astrophysics of Gaseous Nebulae and Active Galactic Nuclei (2nd ed.; Sausalito, CA: Univ. Science Books), 126

Polletta, M., Tajer, M., Maraschi, L., et al. 2007, ApJ, 663, 81

Richards, G. T., Lacy, M., Storrie-Lombardi, L. J., et al. 2006, ApJS, 166, 470

Röllig, M., Abel, N. P., Bell, T., et al. 2007, A\&A, 467, 187

Ross, N. P., Hamann, F., Zakamska, N. L., et al. 2015, MNRAS, 453, 3932

Sigut, T. A. A., \& Pradhan, A. K. 2003, ApJS, 145, 15

Tristram, K. R. W., Burtscher, L., Jaffe, W., et al. 2014, A\&A, 563, A82

Tristram, K. R. W., Meisenheimer, K., Jaffe, W., et al. 2007, A\&A, 474, 837

Trump, J. R., Hall, P. B., Reichard, T. A., et al. 2006, ApJS, 165, 1

Turnshek, D. A., Weymann, R. J., Liebert, J. W., Williams, R. E., \& Strittmatter, P. A. 1980, ApJ, 238, 488

Urrutia, T., Becker, R. H., White, R. L., et al. 2009, ApJ, 698, 1095

Veilleux, S., Trippe, M., Hamann, F., et al. 2013, ApJ, 764, 15

Verner, E. M., Verner, D. A., Korista, K. T., et al. 1999, ApJS, 120, 101

Voit, G. M., Weymann, R. J., \& Korista, K. T. 1993, ApJ, 413, 95

Wang, H., Xing, F., Zhang, K., et al. 2013, ApJL, 776, L15

Wang, T., Zhou, H., Yuan, W., et al. 2009, ApJ, 702, 851

Wang, T. G., Yang, C. W., Wang, H. Y., \& Ferland, G. J. 2015, ApJ, 814, 150

Weymann, R. J., Morris, S. L., Foltz, C. B., \& Hewett, P. C. 1991, ApJ, 373, 23

Zhang, K., Dong, X.-B., Wang, T.-G., \& Gaskell, C. M. 2011, ApJ, 737, 71

Zhang, S., Wang, H., Wang, T., et al. 2014, ApJ, 786, 42

Zhang, S., Wang, T.-G., Wang, H., et al. 2010, ApJ, 714, 367

Zhang, S., Zhou, H., Wang, T., et al. 2015, ApJ, 803, 58 